EPJ Web of Conferences 67, 02065 (2014)

DOI: $10.1051 /$ epjconf / 20146702065

(C) Owned by the authors, published by EDP Sciences, 2014

\title{
Evaluation and accuracy of the local velocity data measurements in an agitated vessel
}

\author{
Bohuš Kysela $^{1 \mathrm{a}}$, Jiří Konfršst ${ }^{1}$, Zdeněk Chára ${ }^{1}$, and Michal Kotek ${ }^{2}$ \\ 1 Institute of Hydrodynamics, AS CR, v.v.i. \\ Pod Patankou 30/5,16612 Prague 6, Czech Republic \\ 2 Institute for Nanomaterials, Advanced Technology and Innovation, Technical University of Liberec \\ Studentska 1402/2, Liberec, Czech Republic
}

\begin{abstract}
Velocity measurements of the flow field in an agitated vessel are necessary for the improvement and better understanding of the mixing processes. The obtained results are used for the calculations of the impeller pumping capacity, comparison of the power consumption etc. We performed various measurements of the local velocities in an agitated vessel final results of which should be processed for several purposes so it was necessary to make an analysis of the obtained data suitability and their quality. Analysed velocity data were obtained from the LDA (Laser Doppler Anemometry) and PIV (Particle Image Velocimetry) measurements performed on a standard equipment where the flat bottomed vessel with four baffles was agitated by the six-blade Rushton turbine. The results from both used methods were compared. The frequency analyses were examined as well as the dependency of the data rates, time series lengths etc. The demands for the data processed in the form of the ensemble-averaged results were also established.
\end{abstract}

\section{Introduction}

The velocity measurements inside the agitated vessel are used to investigate the fluid flow and its turbulent behaviour during the mixing process. Then this knowledge is the background for better understanding of the mixing processes, scale-up modelling, geometry improvement, etc. Although the development of mixing processes is nowadays based mostly on CFD (Computational Fluid Dynamics) where the LES (Large Eddy Simulation) approach is commonly used e.g. [1], [2], [3], [4], [5], the DNS (Direct Numerical Simulation) approach is also under development [6]. The large part of published results in this field were summarised in [7], [8]. The results of the CFD based on the RANS (Reynolds Averaged Navier Stokes) approach were formerly validated by the measurements of the mean values. The fast improvement in the CFD requires the much higher quality of the measured data which should be with high resolution in time and space. The PIV method seems to be the fine instrument and allows the detailed flow analysis [9]. But the high time and space resolution reduces the final time record length. Hence, we compared the velocities obtained by PIV method with LDA measurements, where the LDA measurements were performed in long time series, which was longer than $360 \mathrm{~s}$, while the PIV data records were $10 \mathrm{~s}$. The LDA measurements have been used by many investigators e.g. [10], [11], [12], [13], [14], [15] and as well as the PIV measurements e.g. [16], [17], [18]. In the most of above cited experiments, the cylindrical vessel with standard Rushton impeller had been used [10], [13], [17], [18]. The same trend is also in CFD [8], therefore we used similar equipment with standard Rushton impeller. The radial velocity profiles were obtained and the

\footnotetext{
a e-mail: kysela@ih.cas.cz
}

values of the mean ensemble-averaged velocity and fluctuation velocity in the impeller outflow stream were investigated.

\section{Experimental}

Measurements of the velocity profiles were carried out in a flat bottomed mixing vessel, with water as the working liquid (density $\rho=1000 \mathrm{~kg} \cdot \mathrm{m}^{-3}$, dynamic viscosity $\mu=1 \mathrm{mPa} \cdot s$ ). The vessel was equipped with four radial baffles and agitated with six-blade standard Rushton turbine (see figure 1). The stirred vessel diameter was $T=300 \mathrm{~mm}$, filled with liquid to the level $H=T$. The diameter of the impeller was $D=100 \mathrm{~mm}(T / D=3)$ and installed with off-bottom clearance $C / D=0.75$. The width of baffles was $b=0.1 T$. The measurements were carried out with impeller speed $300 \mathrm{rpm}$, when Reynolds number was $5.0 \cdot 10^{4}$, which represents turbulent regime $\left(\operatorname{Re}>10^{4}\right)$ for agitated vessel. In turbulent regime the mean velocity and fluctuations are dependent only on impeller tip speed $V_{\text {tip }}$ and geometry. Hence, the velocity profiles are depicted in dimensionless form. The positions are also in dimensionless form where $z / H$ is ratio of the total liquid depth and represents vertical position which is parallel with the axis of the impeller shaft. The axis of impeller symmetry is at $z / H=0.283$ where the central disk is. The radial position is expressed by the dimensionless radial component $r^{*}=2 r / D$.

\subsection{LDA measurements}

LDA 1D measurements of the radial velocity in the impeller discharge stream were performed in axial profile with 


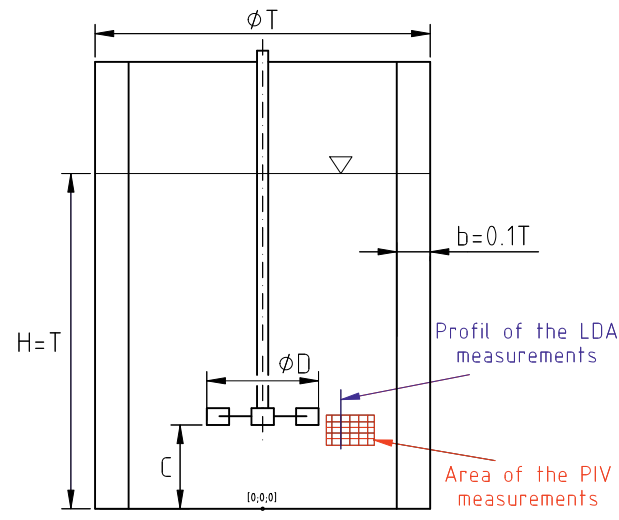

Fig. 1. The pilot plant cylindrical vessel with a six-blade standard Rushton turbine $(\mathrm{T}=300 \mathrm{~mm}, \mathrm{H} / \mathrm{T}=1, \mathrm{C} / \mathrm{D}=0.75, \mathrm{~b} / \mathrm{T}=1 / 10$, four baffles)

selected distance $20 \mathrm{~mm}$ from the impeller blade (figure 1). The value of the dimensionless radial component $r^{*}=2 r / D$ was 1.4. One component LDA system set-up consists of: laser supply Coherent INNOVA 305 Ion-Argon with power $5 \mathrm{~W}$ and separated beam only for one component measurement on wavelength $514.5 \mathrm{~nm}$, DANTEC fibreflow transmitting optics and P80 DANTEC BSA processor. The setup was supervised by BSA FLOW SOFTWARE v3.0 installed on PC where the data was processed. S-HGS (Silver coated - Hollow Glass Spheres) with the mean diameter $10 \mu \mathrm{m}$ and density $1.1 \mathrm{~g} \cdot \mathrm{cm}^{-3}$ were used as trace particles.

\subsection{PIV measurements}

The results from region under the impeller were obtained by the PIV measurement technique. The investigated area was $45 \times 30 \mathrm{~mm}$ (figure 1). The area was adjusted $3 \mathrm{~mm}$ above the impeller axis of symmetry and $5 \mathrm{~mm}$ from the impeller blade (figure 1). The PIV system was time-resolved LITRON LDY 304, Nd:YLF laser; high speed PIV-regime camera SpeedSence 611 (with full resolution 1280x800) supplied by the DANTEC. Rhodamine B fluorescent particles with the mean diameter $10 \mu \mathrm{m}$ were used as seeding particles along with wavelength filter to reduce laser reflections. That system was supervised by the Dynamic Studio software which was also used for velocity evaluation. The final evaluated vector grid was $82 \times 47$. The operating frequency was $1 \mathrm{kHz}$ (that means 1000 vector fields per second) the record length was $10 \mathrm{~s}$.

\section{Results}

The results of the obtained velocity profiles of the radial velocity component from both methods PIV and LDA are depicted in figure 2 where the mean ensemble-averaged velocity is normalised by the impeller tip speed. The root mean values of the fluctuating velocity normalised by the impeller tip speed velocity are shown in figure 3. In figures 2 and 3 are depicted points where the deeper analysis was performed. The main parameters of data points are summarised in table 1 for LDA measurements ( 3 points) and in table 2 for PIV measurements ( 2 points).

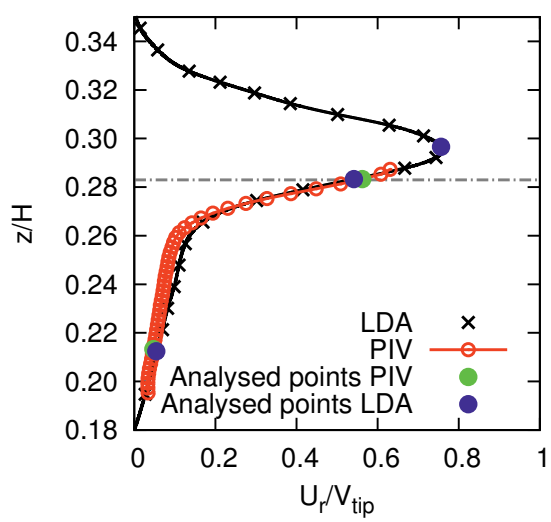

Fig. 2. Mean ensemble-averaged radial velocity measured by both LDA (360 s) and PIV (10 s) method.

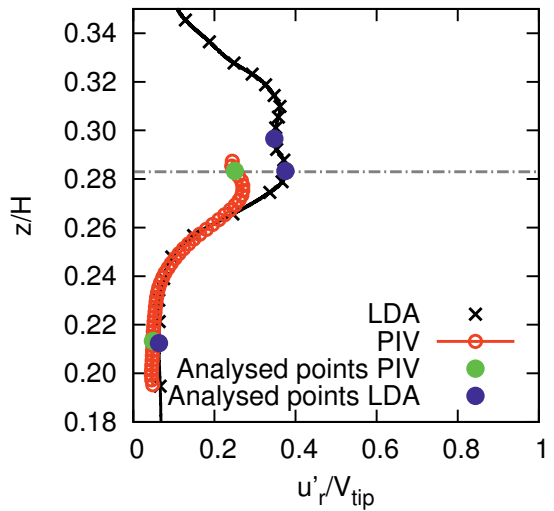

Fig. 3. Root mean square values of the radial fluctuation velocity obtained from both LDA and PIV measurements.

Table 1. Selected points from LDA measurements depicted on profiles in figures 2 and 3.

\begin{tabular}{ccc}
\hline$r / H$ & $U_{r}(\mathrm{~m} / \mathrm{s})$ & $u_{r}^{\prime}(\mathrm{m} / \mathrm{s})$ \\
\hline 0.297 & 1.187 & 0.546 \\
0.283 & 0.850 & 0.589 \\
0.212 & 0.085 & 0.100 \\
\hline
\end{tabular}

Table 2. Selected points from PIV measurements depicted on profiles in figures 2 and 3.

\begin{tabular}{ccc}
\hline$r / H$ & $U_{r}(\mathrm{~m} / \mathrm{s})$ & $u_{r}^{\prime}(\mathrm{m} / \mathrm{s})$ \\
\hline 0.283 & 0.883 & 0.393 \\
0.213 & 0.074 & 0.076 \\
\hline
\end{tabular}

\subsection{Velocity record length}

At the first stage the temporal length of the instantaneous velocities data record was compared. The mean values and root mean square values were evaluated from time records obtained by the LDA measurements. The results of the time records 5; 60; $360 \mathrm{~s}$ are shown in figures 4 and 5 . It is obvious that the evaluated results of the ensembleaveraged values are practically the same after $60 \mathrm{~s}$ in each point of the profile. Nevertheless, this conclusion is valid only when the data rate is $120 \mathrm{~Hz}$ and higher (for used im- 


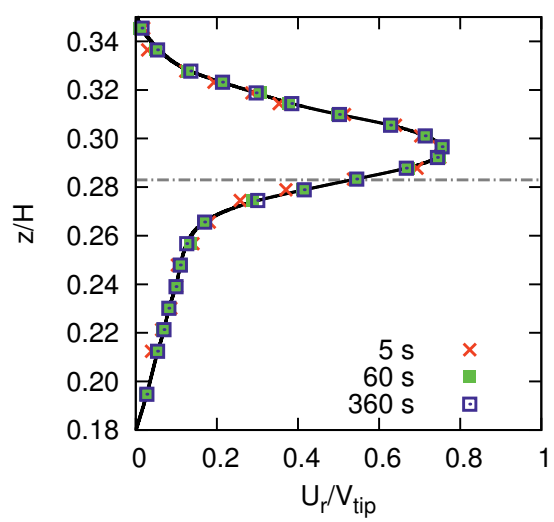

Fig. 4. Mean ensemble-averaged radial velocity evaluated from $5 ; 60 ; 360 \mathrm{~s}$ temporal length of the record at each point of LDA measurement.

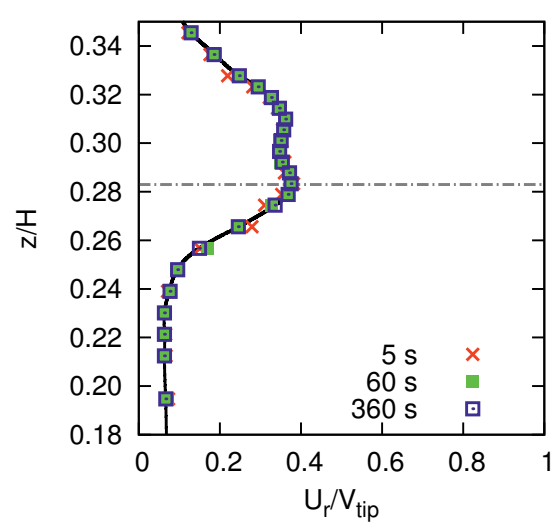

Fig. 5. Root mean square values of the radial fluctuation velocity evaluated from $5 ; 60 ; 360 \mathrm{~s}$ temporal length of the record at each point of LDA measurement.

peller speed) because the data rate has also effect on the averaging process. But the discrepancy is visible for $5 s$ of the record length. The change of the averaged values vs. time record length was evaluated in selected points and it is shown for LDA measurements in figures 6 and 7 for the mean ensemble-averaged velocity or root mean square of fluctuating velocity, respectively. The same relations of the PIV measurements are depicted in figures 8 and 9 where the record length is limited with the maximal value $10 \mathrm{~s}$ of the PIV record. All obtained relations show relatively stable values after $5 s$ of the record, what represents 25 rounds of the impeller at the impeller speed $300 \mathrm{rpm}$ and 5000 averaged values with $1 \mathrm{kHz}$ of the data rate of the PIV measurement. This result is very similar with conditions derived from CFD simulations, where 20 rounds of the impeller are the minimum to obtained stable averaged data of the velocity [1], [3]. The effect of low frequencies did not occur, probably, because the measurements were performed in vicinity of the impeller, where the velocity is high enough.

\subsection{Velocity distribution in selected points}

Because the discrepancy between the root mean square values obtained by the LDA and PIV measurements had not

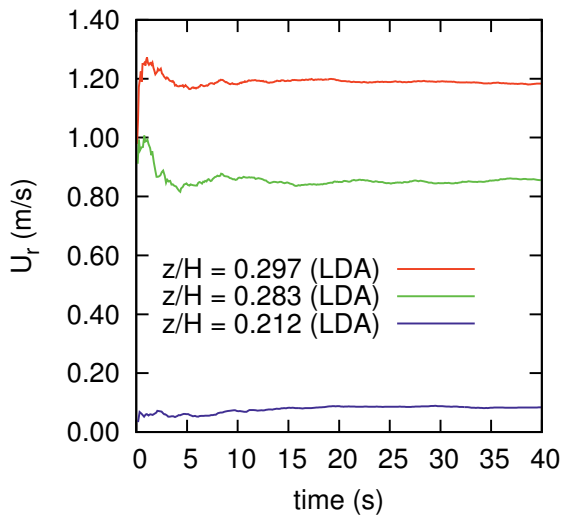

Fig. 6. Mean ensemble-averaged radial velocity vs. temporal length of the record in selected points of LDA measurement (figure 2, table 1).

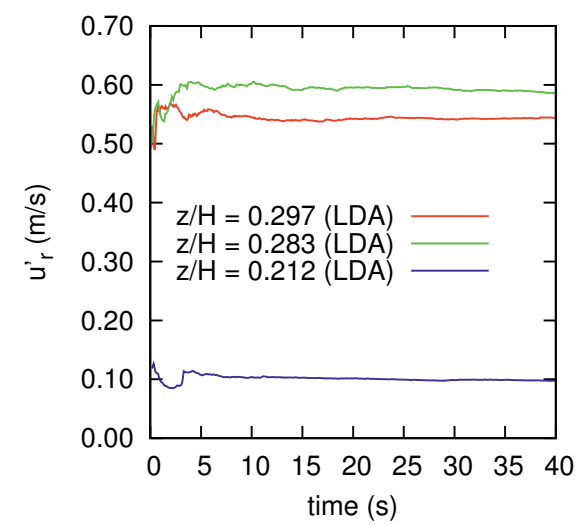

Fig. 7. Root mean square values of the radial fluctuation velocity vs. temporal length of the record in selected points of LDA measurement (figure 3, table 1).

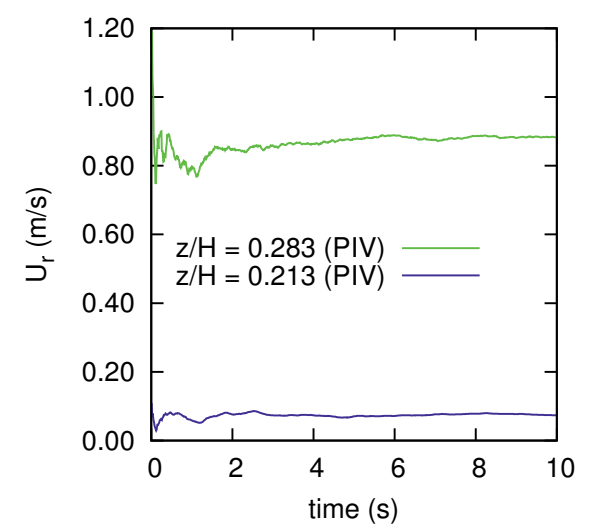

Fig. 8. Mean ensemble-averaged radial velocity vs. temporal length of the record at selected points of PIV measurement (figure 2 , table 1).

been quite explained, the distributions of the velocity at each point were examined. The comparison of histograms in point $z / H=0.283$ shows more wide spectrum of velocities measured by the LDA. It could be caused by the basic principles of measurements. The LDA measures the signal from individual tracking particle which is passing through the measurement volume (laser beams intersection with diameter $\approx 1 \mathrm{~mm}$ ), while the PIV method evaluates the ve- 


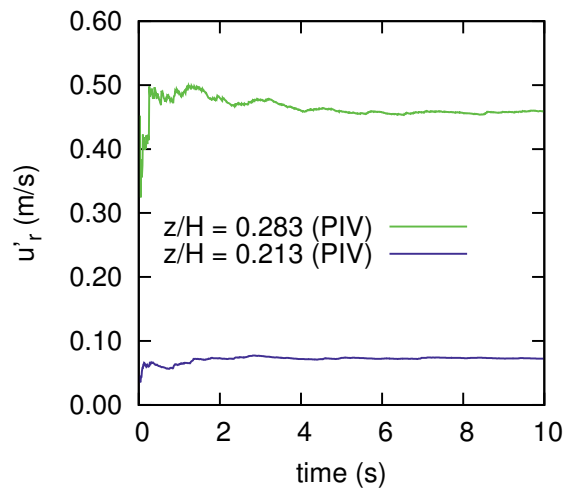

Fig. 9. Root mean square values of the radial fluctuation velocity vs. temporal length of the record at selected points of PIV measurement (figure 3 , table 1 ).

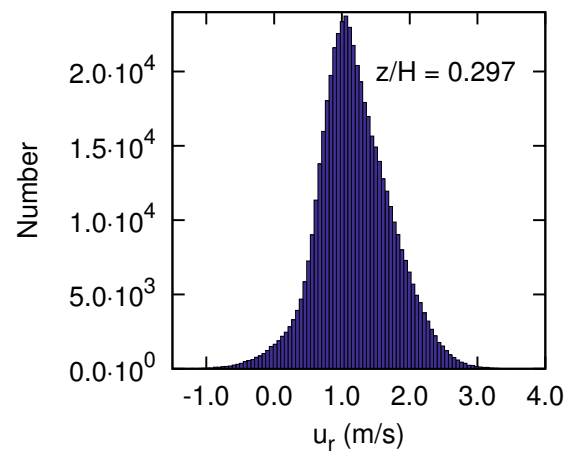

Fig. 10. Histogram of radial velocity measured by LDA at point $\mathrm{z} / \mathrm{H}=0.297$ (table 1).

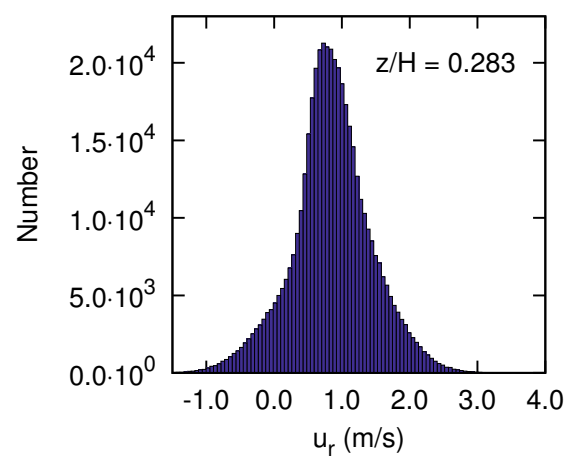

Fig. 11. Histogram of radial velocity measured by LDA at point $\mathrm{z} / \mathrm{H}=0.283$ (table 1).

locity in interrogation area $(0.76 \mathrm{~mm})$, thus the velocities are partly spatially averaged.

\subsection{Frequency analysis}

Because the trailing vortices were readily recognised in PIV results [12], [16], [9] and, moreover, the LDA data are unevenly sampled [19] in contrary with the PIV data, the autocorrelation functions and power spectral density were compared. For the unevenly sampled LDA data the slot correlation technique was used [20],[19]. The power spectra are depicted in figure 15 . The most significant peak

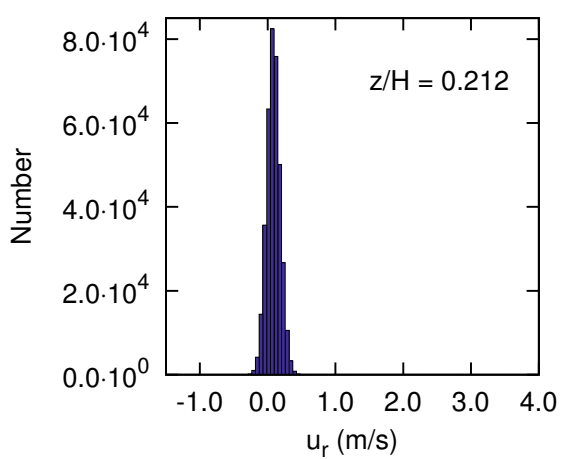

Fig. 12. Histogram of radial velocity measured by LDA at point $\mathrm{z} / \mathrm{H}=0.212$ (table 1).

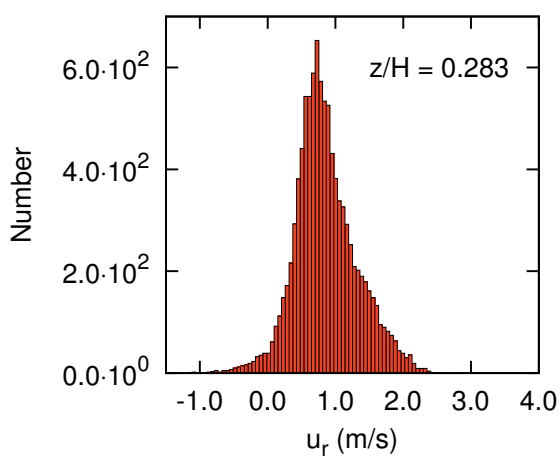

Fig. 13. Histogram of radial velocity measured by PIV at point $\mathrm{z} / \mathrm{H}=0.283$ (table 1$)$.

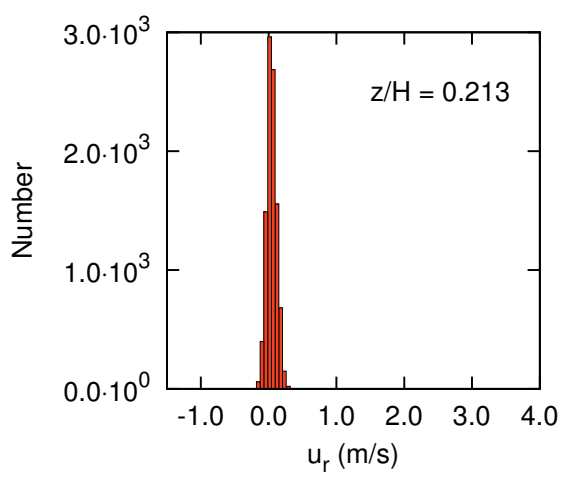

Fig. 14. Histogram of radial velocity measured by PIV at point $\mathrm{z} / \mathrm{H}=0.213$ (table 1).

can be observed at frequency $30 \mathrm{~Hz}$, which corresponds to the impeller speed $5 s^{-1}(300 \mathrm{rpm})$ multiplied by 6 (number of blades). The peak occurring at frequency $60 \mathrm{~Hz}$ is the second-harmonic one. The peak at frequency $5 \mathrm{~Hz}$ is proportional to the oscillations of the primary circulation loop [21] or could be generated by the impeller shaft oscillations as well as by the impeller irregularity. The peak occurring at frequency $10 \mathrm{~Hz}$ is probably the second-harmonic one. Although the conditions of data processing are various for each measurement method, the results of the power spectra are in good agreement in both compared points. 


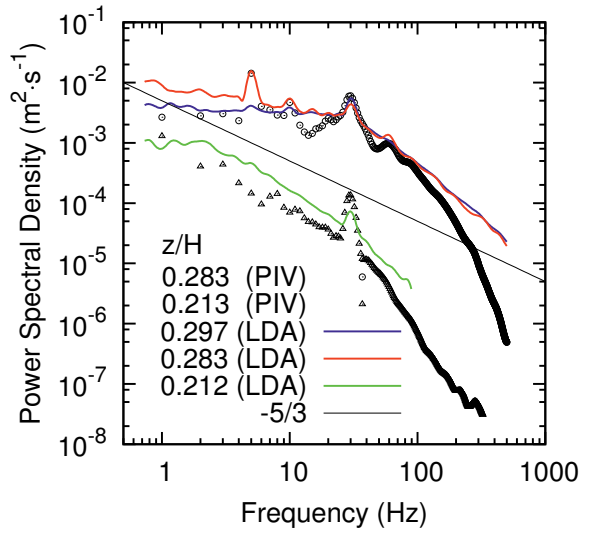

Fig. 15. Comparison of the frequency spectra at selected points (figures 3 and 2, tables 1 and 2).

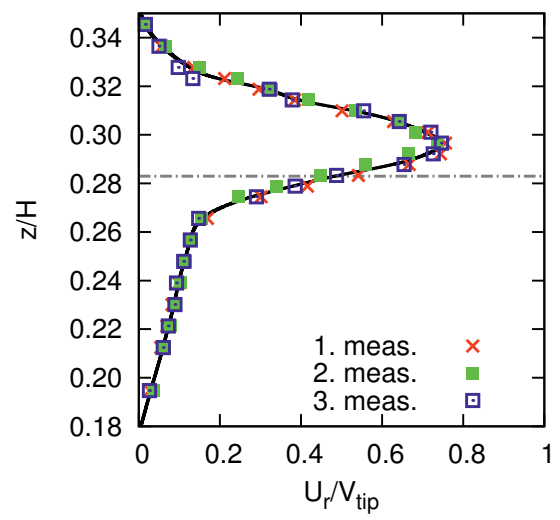

Fig. 16. Comparison of the mean ensemble-averaged radial velocity profiles obtained from three independent LDA measurements.

\subsection{Accuracy and reproducibility}

The reproducibility of the measurements is also important. We performed several independent measurements in an agitated vessel and the three ensemble-averaged results obtained by the LDA method are depicted in figures 16 and 17. The measurements were carried out during the long time but without the rearrangement of the vessel geometry and without the readjusting of the traverse system. It was demonstrated that the correct determination of the position has a big effect on accuracy, namely in the regions with the high velocity gradients.

\section{Conclusions and discussion}

The results of the radial mean ensemble-averaged velocity obtained from the LDA and PIV measurements are in good agreement in the region of the impeller outflow stream. The root mean square values of the fluctuations velocity are lower for PIV measurements in the main radial jet region. The relation between the time record length and the above mentioned root mean square values was not been settled. In contrary, it was shown that the length of the temporal record of the ensemble-averaged velocity values has considerable effect to $10 s$ of the record length in region

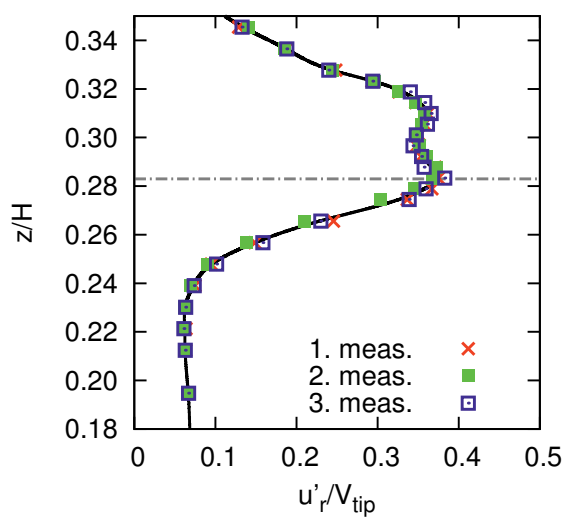

Fig. 17. Comparison of the root mean square values of the radial fluctuation velocity profile obtained from three independent LDA measurements.

of the investigated impeller outflow stream. Subsequently the comparison of the velocity distribution in measured point at the axis of the impeller symmetry shows similar shape, but the distribution is wider for LDA measurements, namely in region where the velocities are higher than impeller tip speed. Nevertheless, the evaluated power spectra are in good agreement at frequencies up to $100 \mathrm{~Hz}$ for both investigated points. Repeated measurements showed very good reproducibility. The LDA measurements have the maximal deviation of the evaluated ensemble-averaged values lower than $5 \%$ in regions with high velocity gradients which is mainly caused by the accuracy of the position determination, in other cases the deviation is lower than $1 \%$, if the position error is sufficiently eliminated.

\section{Symbols}

b baffle width, $\mathrm{m}$

C off-bottom clearance, $m$

D impeller diameter, $\mathrm{m}$

$\mathrm{H}$ total liquid depth, $\mathrm{m}$

$\mathrm{r}^{*} \quad$ dimensionless radius of agitated vessel, -

Re Reynolds number, -

$\mathrm{T}$ vessel diameter, $\mathrm{m}$

$\mathrm{u} \quad$ fluid velocity, $\mathrm{m} \cdot \mathrm{s}^{-1}$

$\mathrm{u}^{\prime} \quad$ root mean square of fluctuations velocity, $\mathrm{m} \cdot \mathrm{s}^{-1}$

$\mathrm{U}$ mean ensemble-averaged velocity, $\mathrm{m} \cdot \mathrm{s}^{-1}$

\section{Acknowledgments}

This research has been subsidized by the research project No. GA ČR P101/12/2274 and RVO: 67985874.

\section{References}

1. J. Derksen, H. Van den Akker, AICHE JOURNAL 45, 209 (1999)

2. M. Jahoda, M. Mostek, A. Kukukova, V. Machon, Chemical Engineering Research and Design 85, 616 (2007) 
3. Z. Li, Y. Bao, Z. Gao, Chemical Engineering Science 66, 1219 (2011)

4. Z. Li, M. Hu, Y. Bao, Z. Gao, Industrial \& Engineering Chemistry Research 51, 2438 (2012)

5. J. Gimbun, C.D. Rielly, Z.K. Nagy, J.J. Derksen, AIChE Journal 58, 3224 (2012)

6. J.J.J. Gillissen, H.E.A. Van den Akker, AIChE Journal 58, 3878 (2012)

7. J.B. Joshi, N.K. Nere, C.V. Rane, B.N. Murthy, C.S. Mathpati, A.W. Patwardhan, V.V. Ranade, The Canadian Journal of Chemical Engineering 89, 23 (2011)

8. J.B. Joshi, N.K. Nere, C.V. Rane, B.N. Murthy, C.S. Mathpati, A.W. Patwardhan, V.V. Ranade, The Canadian Journal of Chemical Engineering 89, 754 (2011)

9. A. Line, J.C. Gabelle, J. Morchain, D. Anne-Archard, F. Augier, Chemical Engineering Research and Design pp. - (2013)

10. H. Wu, G. Patterson, Chemical Engineering Science 44, 2207 (1989)

11. S.M. Kresta, P.E. Wood, Chemical Engineering Science 48, 1761 (1993)

12. M. Schafer, M. Hofken, F. Durst, Chemical Engineering Research and Design 75, 729 (1997), fluid Flow

13. K.C. Lee, M. Yianneskis, AIChE Journal 44, 13 (1998)

14. B.C. Venneker, J.J. Derksen, H.E.V. den Akker, Chemical Engineering Research and Design 88, 827 (2010)

15. I. Fort, B. Kysela, T. Jirout, Chemical and Process Engineering - Inzynieria Chemiczna i Procesowa 31, 661 (2010)

16. V. Ranade, M. Perrard, N.L. Sauze, C. Xuereb, J. Bertrand, Chemical Engineering Research and Design 79, 3 (2001)

17. K.V. Sharp, R.J. Adrian, AIChE Journal 47, 766 (2001)

18. R. Escudie, A. Line, AIChE Journal 49, 585 (2003)

19. B. Kysela, J. Konfrst, Z. Chara, LDA measurements and turbulence spectral analysis in an agitated vessel, in EFM12 - EXPERIMENTAL FLUID MECHANICS 2012, edited by Dancova, P and Novonty, P (2013), Vol. 45 of EPJ Web of Conferences, ISBN 978-807372-912-7

20. H. Nobach, Experiments in Fluids 32, 337 (2002), 10.1007/s003480100362

21. O. Bruha, T. Bruha, I. Fort, M. Jahoda, Acta Polytechnica 47, 17 (2007) 